\title{
Aspectos relacionados à presença de fungos toxigênicos em uvas e ocratoxina A em vinhos
}

\author{
Aspects related to the presence of toxigenic fungi in grapes and ochratoxin $A$ in wines
}

\author{
Juliane Elisa Welke ${ }^{I^{*}}$ Michele Hoeltz $^{\mathrm{I}}$ Isa Beatriz Noll ${ }^{\mathrm{I}}$
}

- REVISÃo BIBLIOGRÁFICA -

\section{RESUMO}

A colonização dos Aspergillus da secção Nigri nas uvas durante o cultivo é a principal fonte de ocratoxina A (OTA) nos vinhos. A. carbonarius e A. niger são os principais produtores desta micotoxina em uvas e são fungos oportunistas que, se desenvolvem, principalmente, nas bagas danificadas durante seu amadurecimento. A produção de OTA em uvas é influenciada pelas condições climáticas e áreas geográficas, bem como pela variedade de uva, pelo sistema de cultivo e pelos danos causados nas uvas por insetos, infecção fúngica ou excesso de irrigação e chuva. As medidas para o controle de fungos toxigênicos devem considerar esses pontos críticos de controle. A OTA presente nas uvas é transferida para o vinho durante o processo de vinificação, sendo que um aumento na concentração de OTA ocorre após a maceração das uvas. Durante o envelhecimento do vinho, observa-se que a toxina permanece estável, pois a mesma concentração de OTA é encontrada no vinho após um ano de armazenamento. Boas práticas de produção, como, por exemplo, a seleção e separação dos cachos de uva com desenvolvimento fúngico visível auxilia, consideravelmente, na redução dos níveis de contaminação por fungos produtores de OTA, bem como dos níveis dessa micotoxina nos vinhos.

Palavras-chave: Ocratoxina A, uva, vinho, Aspergillus.

\section{ABSTRACT}

The infection of grapes by 'black aspergilli' in the field is the main source of ochratoxin A (OTA) in the wine. Aspergillus carbonarius and A. niger fungi are the main producers of this mycotoxin in grapes. They are opportunistic fungi that develop mainly on damaged berries at ripening. The production of OTA in grapes is influenced by climatic conditions, geographical location, grape varieties, crop system, berries damage caused by insets, fungal infection or excessive irrigation and rainfall. Control measures for toxigenic mycoflora in the vineyards must consider these critical control points. OTA in grapes is transferred to wine during vinification and an increase of OTA concentration in must was observed during maceration. The toxin remains stable during wine aging because the same OTA concentration is found in wine after 1 year. Good Agriculture Practices including balanced soil tillage, irrigation, nitrogen fertilization and pruning associated with Good Manufacturing Practices, such as separation of rot bunches helps considerably to reduce OTA-producing fungi and levels of mycotoxin in wine.

Key words: Ochratoxin A, grape, wine, Aspergillus.

\section{INTRODUÇÃO}

Os fungos são capazes de produzir, em condições naturais e laboratoriais, compostos que não são essenciais para seu crescimento e sobrevivência, chamados metabólitos secundários. As micotoxinas são metabólitos secundários tóxicos produzidos por fungos filamentosos que ocorrem naturalmente como contaminantes de produtos agrícolas e demonstram propriedades tóxicas em animais quando administradas por via natural, essencialmente por via oral (ABRAMSON, 1998). Entretanto, o desenvolvimento dos fungos não está necessariamente associado com a produção de micotoxinas. O potencial micotoxigênico do fungo depende largamente da cepa, bem como da

Instituto de Ciência e Tecnologia de Alimentos, Universidade Federal do Rio Grande do Sul (UFRGS), 91570-901, Porto Alegre, RS, Brasil. E-mail: juliwelke@yahoo.com.br.*Autor para correspondência. 
composição física e química da matriz e de fatores ambientais (DRUSCH \& RAGAB, 2003). As micotoxinas de maior importância são produzidas principalmente por espécies dos gêneros Aspergillus, Penicillium e Fusarium. A ocratoxina A(OTA) foi uma das primeiras micotoxinas a ser descoberta, sendo isolada e identificada a partir de uma cultura de Aspergillus ochraceus por VAN DER MERWE (1965).

A OTA tem sido detectada em diversos tipos de alimentos, incluindo trigo (RIBA et al., 2008), milho (SEKIYAMA et al., 2005; MAGNOLI et al., 2007), café (LEONI et al., 2000; LEONG et al., 2007), cacau (MOUNJOUENPOU et al., 2008), cevada (VISWANATH et al., 2007), cerveja (KAAASHIMA et al., 2007), figos secos (KARBANCIOLU \& HEPERKAN, 2008), queijo (DALL'ASTA et al., 2008), centeio (JORGENSEN \& JACOBSEN, 2002), pão (ZINEDINE et al., 2007), uva (LASRAM et al., 2007) e produtos derivados, incluindo suco e vinho (BURDASPAL \& LEGARDA, 2007). Na Europa, depois dos cereais, o vinho é a maior fonte de OTA ingerida pela população (MIRAGLIA \& BRERA, 2002).

O objetivo desta revisão bibliográfica é apresentar os aspectos relacionados à presença de fungos toxigênicos em uvas, a ocorrência e as espécies fúngicas envolvidas na produção desta micotoxina em diversos países, os fatores que influenciam a contaminação dos vinhos por ocratoxina $\mathrm{A}$ e a sua ocorrência.

Aspectos toxicológicos da ocratoxina A

A OTA é uma isocoumarina ligada a uma molécula de fenilalanina que possui diversas propriedades tóxicas, notavelmente nefrotóxicas (JECFA, 2001). Esta micotoxina foi associada à nefropatia endêmica dos Balcãs, doença caracterizada por progressiva redução das funções renais em humanos. Em estudos com animais, a OTA tem demonstrado efeitos carcinogênicos (BROWN et al., 2007), mutagênicos (PALMA et al., 2007), teratogênicos (BALASAHEB et al., 2007), imunossupressores (ROSSIELLO et al., 2008), genotóxicos (TOZLOVANU et al., 2006) e neurotóxicos (SAVA et al., 2006). Em virtude da evidente carcinogenicidade em animais, a OTA foi classificada pela Agência Internacional de Pesquisa sobre o Câncer (IARC) no grupo 2B, ou seja, como possivelmente carcinogênica para humanos (IARC, 1993).

A OTA foi encontrada em sangue humano em todos os países onde foi investigada, frequentemente em alta incidência, como, por exemplo, em Portugal (LINO et al., 2008), na Tunísia (HASSEN et al., 2004), na Costa do Marfim (SANGARE-TIGORI et al., 2006), na Noruega e na Suíça (THUVANDER et al., 2001). A principal preocupação, contudo, nos níveis em que é normalmente detectada no sangue das populações, deve-se a suas propriedades imunotóxicas, pois a OTA apresenta esse efeito em concentrações da ordem de $n g \mathrm{~mL}^{-1}$ (LINO et al., 2008).

O Comitê de Peritos em Aditivos Alimentares da Organização das Nações Unidas para Agricultura e Alimentação (FAO)/Organização Mundial da Saúde (WHO) estabeleceu a ingestão semanal tolerável provisória de 100ng kg-1 de peso corpóreo (p.c) (JECFA, 2001). Com base nas informações toxicológicas e de exposição, a União Européia estabeleceu o limite máximo permitido de $2 \mu \mathrm{g} \mathrm{L}^{-1}$ para OTA em vinho, suco, néctar e mosto de uva (COMMISSION OF THE EUROPEAN COMMUNITIES, 2006).

Espécies de fungos produtoras de ocratoxina A em uvas

Os fungos responsáveis pela acumulação de OTA em cereais, principalmente $\boldsymbol{A}$. ochraceus e $\boldsymbol{P}$. verrucosum, foram inicialmente relacionados à formação dessa micotoxina em uvas. Entretanto, estudos recentes demonstram que as espécies produtoras de OTA mais frequentemente detectadas nas uvas de diversos países pertencem à secção Nigri, mais concretamente ao agregado $\boldsymbol{A}$. niger e $\boldsymbol{A}$. carbonarius (Tabela 1).

A maioria dos investigadores tem constatado dois fatos. Em primeiro lugar, constatou-se que as estirpes do agregado $\boldsymbol{A}$. niger são mais abundantes nas uvas que $\boldsymbol{A}$. carbonarius. Em segundo lugar, constatou-se, entretanto, que A. carbonarius parece ser muito mais relevante em termos de produção de OTA nas uvas, com uma maior proporção de estirpes produtoras da micotoxina, e em níveis mais elevados que as estirpes do agregado $A$. niger (ABARCA et al., 2001; BATTILANI \& PIETRI 2002; ROSA et al., 2002; MAGNOLI et al., 2003; BELLÍ et al., 2004b; OLIVERI et al., 2008; KHOURY et al., 2008).

Ocasionalmente, em uvas, foram detectadas cepas produtoras de OTA de outras espécies de Aspergillus, em particular da secção Circumdati (particularmente A. ochraceus) (ROSA et al., 2002; BATTILANI et al., 2003; BAU et al., 2005). Essas espécies, entretanto, não são relevantes para a contaminação das uvas dada a sua baixa frequência.

Fatores relacionados à contaminação das uvas por OTA Os Aspergillus da secção Nigri causam a chamada podridão preta na uva, que as torna secas e com aspecto enrugado. A incidência da colonização das uvas por Aspergillus é aumentada quando ocorre 
Tabela 1 - Fungos responsáveis pela contaminação de uvas por ocratoxina A em alguns países.

\begin{tabular}{|c|c|c|}
\hline País & Espécie fúngica & Referência \\
\hline Portugal & $\begin{array}{l}\text { A. carbonarius, } A . \quad \text { ochraceus, } \\
\text { agregado A. niger }\end{array}$ & $\begin{array}{l}\text { ABRUNHOSA et al. (2001); SERRA et al. (2003); SERRA et al. } \\
(2006)\end{array}$ \\
\hline França & A. carbonarius & SAGE et al. (2002); LATASTE et al. (2004) \\
\hline Argentina & agregado $\boldsymbol{A}$. niger & ROSA et al. (2002); MAGNOLI et al. (2003) \\
\hline Itália & A. carbonarius, agregado A. niger & BATTILANI et al. (2003); OLIVERI et al. (2008) \\
\hline Espanha & A. carbonarius, A. ochraceus & BELLÍ et al. (2004b); BAU et al. (2005); BELLÍ et al. (2006) \\
\hline Israel & A. carbonarius, agregado A. niger & MITCHELL et al. (2004); GUZEV et al. (2006) \\
\hline Grécia & A. carbonarius, agregado A. niger & TJAMOS et al. (2004); TJAMOS et al. (2006) \\
\hline Austrália & A. carbonarius & LEONG et al. (2004) \\
\hline Hungria & Agregado A. Níger & VARGA et al. (2005) \\
\hline Brasil & $\begin{array}{l}\text { A. carbonarius, } \\
\text { A. ochraceus, agregado A. niger }\end{array}$ & CHULZE et al. (2006) \\
\hline Tunísia & A. carbonarius, agregado A. niger & LASRAM et al. (2007) \\
\hline Líbano & A. carbonarius & KHOURY et al. (2008) \\
\hline
\end{tabular}

irrigação excessiva durante o estágio de maturação das uvas, o que provoca o rompimento das bagas. Períodos de chuva durante a colheita também podem causar o rompimento das bagas, o que favorece a colonização por Aspergillus. Os danos na superfície das bagas causados por insetos, aves e outros fungos também facilitam o desenvolvimento de fungos produtores de OTA(LEONG et al., 2006a).

Quanto à distribuição dos Aspergillus negros em relação aos estágios de desenvolvimento da uva, observou-se que o agregado A. niger é o principal grupo em todas as fases de desenvolvimento das uvas. A incidência de $\boldsymbol{A}$. carbonarius é de duas a três vezes menor que a do agregado $A$. niger, sendo que sua frequência aumenta durante a maturação até a colheita das uvas (BATTILANI et al., 2006).

LEONG et al. (2004) observaram que, em anos chuvosos, ocorreu o favorecimento da infecção por Botrytis e, consequentemente, ocorreu uma maior incidência de Aspergillus negros nas uvas cultivadas na Austrália. Botrytis cinerea é um patógeno causador do mofo cinza em uvas e sua infecção nas uvas leva à abertura de feridas na película das bagas, e favorece a infecção de fungos oportunistas como Aspergillus. No entanto, BATTILANI \& PIETRI (2002) constataram que A. carbonarius é uma espécie muito invasiva, capaz de penetrar nos bagos mesmo sem danos na película, e verificaram que as uvas podem ser susceptíveis à infecção pelo fungo desde os estágios iniciais da maturação. Assim, cachos sem visível desenvolvimento de fungos também podem conter OTA, embora bagas com mofo preto normalmente mostrem níveis mais altos de contaminação.
A Lobesia botrana (Lepidoptera: Tortricidae) é a chamada traça que ataca os cachos em todas as fases do seu desenvolvimento, podendo completar três ou quatro gerações anuais. A primeira geração causa danos às flores, e as gerações seguintes danificam as bagas especialmente durante o amadurecimento. As larvas podem contribuir para a dispersão dos esporos de Aspergillus ou agir como vetores de esporos. Em estudo conduzido por COZZI et al. (2006), as bagas danificadas pela $L$. botrana apresentaram considerável aumento no nível de contaminação pelos Aspergillus negros (acima de $10^{6}$ unidades formadoras de colônias) e acumulação de OTA acima de $1000 \mu \mathrm{g} \mathrm{kg}^{-1}$

Apesar da alta incidência de contaminação de vinhos com OTA, há pouca informação sobre a capacidade que os Aspergillus negros têm de colonizar as bagas e produzir OTA em diferentes variedades de uvas. BATTILANI et al. (2004) inocularam cinco cepas produtoras de OTA em 12 variedades de uvas. As variedades Bianco di Alessano, Pampanuto e Uva di Troia apresentaram baixos níveis da micotoxina, enquanto que a variedade que se mostrou mais susceptível a colonização de Aspergillus foi a Cabernet Sauvignon, com níveis de $200 \mu \mathrm{g} \mathrm{kg}^{-1}$ de OTA.

Diferentes condições climáticas, devido à altitude, podem afetar o desenvolvimento dos fungos produtores de OTA (BATTILANI et al., 2006). MITCHELL et al. (2004) verificaram que a temperatura ótima de crescimento de $\boldsymbol{A}$. carbonarius em meio sintético de composição semelhante ao da uva ocorre entre 30 e $35^{\circ} \mathrm{C}$, sem crescimento a menos de $15^{\circ} \mathrm{C}$, e que a atividade de água (Aa) ótima para crescimento se situava entre 0,93-0,987. Em meio de cultura YES 
(Yeast Extract Sucrose), ESTEBAN et al. (2004) verificaram que cepas de $A$. carbonarius são capazes de crescer entre 10 a $40^{\circ} \mathrm{C}$. Em termos de produção de OTA, verificou-se que os requisitos para a produção da micotoxina eram mais específicos que os exigidos para o crescimento do fungo. As condições ótimas para a produção de OTA variam dependendo da cepa (ESTEBAN et al., 2004; MITCHELL et al., 2004). Algumas cepas produziram níveis máximos de OTA entre 15 e $20^{\circ} \mathrm{C}$ eAa entre 0,95 e 0,98 . A produção máxima de OTA por A. carbonarius foi verificada nos estágios iniciais de crescimento, ao fim de cinco dias de incubação, em meio de composição semelhante à uva (BELLÍ et al., 2004c).

As condições ótimas para o crescimento de fungos do agregado $A$. niger ocorrem entre 35 e $37^{\circ} \mathrm{Ce}$ Aa de 0,93 a 0,98 . A produção de OTA por estes fungos ocorre entre 20 e $25^{\circ} \mathrm{C}$ e Aa de 0,93 a 0,98 (ESTEBAN et al., 2004). Baixa porcentagem de cepas produtoras de OTA (5-10\%) foi detectada entre os fungos do agregado A. niger (PERRONE et al., 2008).

O aumento da temperatura, muitas vezes acima de $30^{\circ} \mathrm{C}$, no mês que precede a colheita das uvas, favorece o desenvolvimento dos Aspergillus negros (BELLÍ et al., 2005). O aumento da umidade relativa entre 80 e $100 \%$ também favorece o desenvolvimento de A. carbonarius, bem como a produção de OTA (BELLÍ et al., 2007). Fungos do agregado A. niger sobrevivem quando as uvas são armazenadas a $0^{\circ} \mathrm{C}$ por 30 dias, enquanto que $\boldsymbol{B}$. cinerea, patógeno que ataca as uvas na pós-colheita, não se desenvolve nessas condições de armazenamento. $\mathrm{O}$ tratamento das uvas com solução de etanol $50 \%$, antes do armazenamento sob refrigeração, eliminou o $B$. cinerea, mas não reduziu o desenvolvimento de $\boldsymbol{A}$. carbonarius observado após o armazenamento. A exposição de uvas naturalmente contaminadas com $\boldsymbol{A}$. carbonarius a $5 p p m$ de $\mathrm{SO}_{2}$ foi suficiente para eliminar o crescimento desses fungos (GUZEV et al., 2008).

\section{Ocratoxina A em vinhos}

A OTA foi detectada pela primeira vez em vinhos da Suíça por ZIMMERLI \& DICK (1996). Desde então, várias pesquisas têm sido conduzidas em relação à ocorrência dessa toxina no vinho. Em países da Europa, vários estudos têm sido realizados para mapear áreas de risco e apontar os pontos críticos de controle, o que auxilia na prevenção e controle dos níveis de OTA nas uvas. Esta micotoxina tem se tornado um problema principalmente para o sul da Europa (ZIMMERLI \& DICK, 1996; OTTENEDER \& MAJERUS, 2000, CABAÑES et al., 2002).
A principal fonte de OTA nos vinhos é a colonização dos Aspergillus da secção Nigri nas uvas durante o cultivo. AOTA presente nas uvas é transferida para o vinho durante o processo de vinificação. Os resultados de pesquisas realizadas em vários países estão relatados na tabela 2. Entre as 2.538 amostras de vinho analisadas, verifica-se uma alta incidência de contaminação, de 41 a $100 \%$. Vinhos analisados no Brasil mostraram frequência de contaminação por OTA de $31 \%$, enquanto que, em países da Europa, como, por exemplo, na Espanha, a contaminação chega a $99 \%$.

Uma correlação positiva entre os níveis de OTA e resveratrol em vinhos foi demonstrada por PERRONE et al. (2007). Os autores sugeriram que os níveis tóxicos de OTA podem ser contrabalançados pelos efeitos benéficos de derivados do resveratrol.

Fatores que influenciam na contaminação de vinhos por ocratoxina $\mathrm{A}$

Com relação à contaminação dos vinhos com OTA de acordo com a região geográfica de cultivo das uvas, a análise dos resultados publicados na literatura permite concluir que há áreas vitivinícolas onde o risco de contaminação é particularmente elevado. Na Itália, PIETRI et al. (2001) verificaram que a origem geográfica dos vinhos influenciava nos níveis de contaminação. Os vinhos produzidos no sul da Itália, onde as condições climáticas são mais favoráveis para o crescimento de fungos produtores de OTA nas uvas, geralmente mostram incidência e níveis de contaminação mais elevados do que os vinhos produzidos no norte e na região central da Itália. A média da concentração de OTA nos vinhos oriundos do noroeste, nordeste, centro e sul de Itália são de 0,$011 ; 0,081 ; 0,295$ e $1,233 \mu \mathrm{g} \mathrm{L}^{-1}$, respectivamente. Por outro lado, BELLÍ et al. (2004a) analisaram vinhos provenientes de regiões da costa Mediterrânica da Espanha e da região de Rioja. Os investigadores detectaram OTA em níveis elevados em vinhos tintos oriundos da região de Rioja (máximo 3,19 $\mu \mathrm{g} \mathrm{L}^{-1}$ ), mas não detectaram relação ou diferenças estatísticas significativas entre a origem, a cor do vinho e os níveis de OTA das amostras. O valor de OTA mais elevado foi detectado em uma amostra de moscatel $\left(15,25 \mu \mathrm{g} \mathrm{L}^{-1}\right)$. Em países do norte de África, foram detectados teores de OTA semelhantes aos que são referidos para países do sul da Europa, o que permite definir a bacia mediterrânea como uma área de risco (FILALI et al., 2001; MARKAKI et al., 2001). A OTA foi detectada também em países como África do Sul (SHEPHARD et al., 2003), Austrália (HOCKING et al., 2003) e Brasil (ROSA et al., 2004), mas em níveis mais baixos que o valor de $2 \mu \mathrm{g} \mathrm{L}^{-1}$ proposto como limite máximo de OTA em vinho pela Comissão da Comunidade Européia. 
Tabela 2 - Ocorrência de ocratoxina A em vinhos produzidos em alguns países.

\begin{tabular}{|c|c|c|c|c|}
\hline Origem & $\mathrm{N}^{*}$ & Incidência OTA (\%) & Variação OTA $\left(\mu \mathrm{g} \mathrm{L}^{-1}\right)$ & Referência \\
\hline Croácia & 10 & 8 & $<0,005-44$ & FLAJS et al. (2009) \\
\hline Europa & 121 & 49 & $<0,024-27,74$ & VALERO et al. (2008) \\
\hline Itália & 1166 & 36 & $<0,01-7,5 \mathrm{ng} \mathrm{L}^{-1}$ & BRERA et al. (2008) \\
\hline Turquia & 95 & 86 & $<0,006-0,815$ & VAR \& KABAK (2007) \\
\hline Espanha & 188 & 99 & $<0,01-4,63$ & BURDASPAL \& LEGARDA (2007) \\
\hline Brasil & 29 & 31 & $<0,1-1,33$ & SHUNDO et al. (2006) \\
\hline Líbano & 42 & 60 & $<0,012-0,126$ & KHOURY et al. (2006) \\
\hline Brasil & 57 & 25 & $<0,03-0,07 \mathrm{ng} \mathrm{L}^{-1}$ & ROSA et al. (2004) \\
\hline Itália & 150 & 80 & $<0,01-5,2$ & FINOLI et al. (2004) \\
\hline Austrália & 601 & 15 & $<0,02-1,0$ & HOCKING et al. (2003) \\
\hline Grécia & 35 & 63 & $<0,02-3,2$ & SOUFLEROS et al. (2003) \\
\hline África do Sul & 24 & 100 & $0,04-0,38$ & SHEPHARD et al. (2003) \\
\hline Itália & 111 & 82 & $<0,001-3,8$ & PIETRI et al. (2001) \\
\hline Europa & 420 & 48 & $<0,01-3,3$ & OTTENEDER \& MAJERUS (2000) \\
\hline Itália & 55 & 87 & $<0.01-7.6$ & VISCONTI et al. (1999) \\
\hline Espanha & 192 & 82 & $<0.01-0.6$ & BURDASPAL \& LEGARDA (1999) \\
\hline Japão & 46 & 41 & $<0.01-0.2$ & UENO (1998) \\
\hline Europa & 118 & 70 & $<0.005-0.4$ & ZIMMERLI \& DICK (1996) \\
\hline
\end{tabular}

* número de amostras analisadas

ZIMMERLI \& DICK (1996) sugerem que as diferenças quanto à região de origem dos vinhos poderiam ser devidas a duas causas possíveis: i) diferente incidência de fungos produtores de OTA nas uvas, sendo que as condições climáticas no sul favorecem o crescimento de espécies de Aspergillus produtoras de OTA; ii) diferentes práticas usadas no cultivo de uvas, como, por exemplo, uso de praguicidas, diferentes variedades cultivadas e diferentes processos de vinificação, relacionados ao tempo e condições de armazenamento das uvas, tipo de maceração, duração e temperatura da fermentação. Os investigadores lançam ainda uma terceira hipótese: a de que os fungos produtores de OTA poderiam crescer nos barris, tanques ou outros equipamentos antes de estar em contacto com as uvas, mosto ou vinho.

Durante o processo de vinificação, a maceração das uvas contribui significativamente para o aumento dos níveis de OTA nos vinhos tintos (FERNANDES et al., 2003). A clarificação através do uso de gelatina contribui para a remoção de $58 \%$ da OTA presente no vinho (LASRAM et al., 2008). LEONG et al. (2006b) relataram que $24 \%$ da OTA originalmente presente nas uvas maceradas foi transferida para o mosto e uma redução de $72 \%$ nos níveis desta micotoxina foram observados após a primeira trasfega (separação da borra). GRAZIOLI et al. (2006) encontraram pouca ou nenhuma redução na concentração de OTA no vinho após a primeira trasfega, enquanto uma redução significativa da OTA foi observada após fermentação maloláctica espontânea, ocorrida entre a primeira e a segunda trasfega. A toxina se mantém estável durante o envelhecimento, pois a mesma concentração de OTA foi encontrada no vinho após um ano (LEONG et al., 2006b). Quanto ao tipo de vinho, os níveis mais elevados de OTA são detectados em amostras de vinhos doces e tintos (ZIMMERLI \& DICK, 1996; HOCKING et al., 2003).

Os dados publicados a respeito dos efeitos do processamento sobre os níveis de OTA são controversos. Isso ocorre devido à impossibilidade do uso de uvas naturalmente contaminadas nos estudos, pois em algumas pesquisas são utilizadas ou uvas inoculadas artificialmente com cepas toxigênicas de $\boldsymbol{A}$. carbonarius (LEONG et al., 2006b), ou uvas adicionadas de OTA (FERNANDES et al., 2003; LASRAM et al., 2008). Essas pesquisas podem não reproduzir as condições naturais de desenvolvimento dos fungos e produção da micotoxina.

\section{CONCLUSÕES}

A principal fonte de contaminação por OTA na produção vitivinícola é a infecção das uvas durante o cultivo pelos Aspergillus da secção Nigri, sendo que o A. carbonarius é a principal espécie identificada como responsável pela produção de OTA. A produção desta micotoxina em uvas é influenciada pelas 
condições climáticas e áreas geográficas, bem como pela variedade de uva, pelo sistema de cultivo e pelos danos causados nas uvas por insetos, infecção fúngica ou excesso de irrigação e chuva. Boas práticas de produção, o que inclui a seleção e separação dos cachos com desenvolvimento fúngico visível, auxiliam consideravelmente na redução dos níveis de contaminação por fungos produtores de OTA, bem como dos níveis desta micotoxina nos produtos derivados.

\section{REFERÊNCIAS}

ABARCA, M.L. et al. Current importance of Ochratoxin Aproducing Aspergillus spp. Journal of Food Protection, Des Moines, v.64, p.903-906, 2001.

ABRAMSON, D. Factors in mycotoxin formation. In: SINHA, K.K.; BHATNAGAR, D. Mcotoxins in agriculture and food safety. New York: Marcel Dekker, 1998. p.255-277.

ABRUNHOSA, L. et al. Mycotoxin production from fungi isolated from grapes. Letters in Applied Microbiology, Oxford, v.32, p.240-242, 2001. Disponível em: <http:// dx.doi.org/10.1046/j.1472-765X.2001.00897.x>. Acesso em: 20 set. 2008 . doi: 10.1046/j.1472-765X.2001.00897.x.

BALASAHEB, W.P. et al. Teratogenic effects of ochratoxin A and aflatoxin B1 alone and in combination on postimplantation rat embryos in culture. Journal of the Turkish German Gynecology Association, Ártemis, v.8, n.4, p. 357364, 2007.

BATTILANI, P.; PIETRI, A. Ochratoxin A in grapes and wine. European Journal of Plant Pathology, Dordrecht, v.108, p.639-643, 2002.

BATTILANI, P. et al. Occurrence of ochratoxin A-producing fungi in grapes grown in Italy. Journal of Food Protection, Des Moines, v.66, p.633-636, 2003.

BATTILANI, P. et al. Ochratoxin A production by Aspergillus carbonarius on some grape varieties grown in Italy. Journal of the Science of Food and Agriculture, London, v.84, p.1736-1740, 2004. Disponível em: <http://dx.doi.org/ 10.1002/jsfa.1875>. Acesso em: 20 set. 2008. doi: 10.1002/ jsfa. 1875 .

BATTILANI, P. et al. Mapping of Aspergillus Section Nigri in Southern Europe and Israel based on geostatistical analysis. International Journal of Food Microbiology, Amsterdam, v.111, n.1, p.72-82, 2006. Disponível em: <http://dx.doi.org/ 10.1016/j.ijfoodmicro.2006.03.014>. Acesso em: 20 set. 2008. doi: $10.1016 /$ j.ijfoodmicro.2006.03.014.

BAU, M. et al. Ochratoxigenic species from Spanish wine grapes. International Journal of Food Microbiology, Amsterdam, v.98, p.125-130, 2005. Disponível em: <http:// dx.doi.org/10.1016/j.ijfoodmicro.2004.05.015>. Acesso em: 20 set. 2008 . doi: $10.1016 /$ j.ijfoodmicro.2004.05.015.

BELLÍ, N. et al. Ochratoxin A in wines, musts and grape juices from Spain. Journal of the Science of Food and Agriculture,
London, v.84, p.591-594, 2004a. Disponível em: <http:// dx.doi.org/10.1016/10.1002/jsfa.1702>. Acesso em: 20 set. 2008. doi: $10.1002 /$ jsfa. 1702 .

BELLÍ, N. et al. Occurrence of ochratoxin A and toxigenic potential of fungal isolates from Spanish grapes. Journal of the Science of Food and Agriculture, London, v.84, p.541546, 2004b. Disponível em: <http://dx.doi.org/10.1016/ 10.1002/jsfa.1658>. Acesso em: 20 set. 2008. doi: 10.1002/ jsfa. 1658 .

BELLÍ, N. et al. Incubation time and water activity effects on ochratoxin A production by Aspergillus section Nigri strains isolated from grapes. Letters in Applied Microbiology, Oxford, v.38, p.72-77, 2004c. Disponível em: <http:// dx.doi.org/10.1046/j.1472-765X.2003.01445.x>. Acesso em: 20 set. 2008 . doi: 10.1046/j.1472-765X.2003.01445.x .

BELLÍ, N. et al. Aspergillus carbonarius growth and ochratoxin A production on a synthetic grape medium in relation to environmental factors. Journal of Applied Microbiology, Oxford, v.98, p.839-844, 2005. Disponível em: <http:// dx.doi.org/10.1046/10.1111/j.1365-2672.2004.02469. $>$. Acesso em: 20 set. 2008. doi: 10.1111/j.13652672.2004.02469.x.

BELLÍ, N. et al. Mycobiota and ochratoxin A producing fungi from Spanish wine grapes. International Journal of Food Microbiology, Amsterdam, v.111, p.S40-S45, 2006. Disponível em: <http://dx.doi.org/10.1016/j.ijfoodmicro.2006.03.011>. Acesso em: 20 set. 2008. doi: 10.1016/j.ijfoodmicro.2006.03.011.

BELLÍ, N. et al. Skin damage, high temperature and relative humidity as detrimental factors for Aspergillus carbonarius infection and ochratoxin A production in grapes. Food Control, Guildford, v.18, n.11, p.1343-1349, 2007. Disponível em: $<$ http://dx.doi.org/10.1016/j.foodcont.2006.02.014>. Acesso em: 20 set. 2008 . doi: 10.1016/j.foodcont.2006.02.014.

BRERA, C. et al. Ochratoxin A contamination in Italian wine samples and evaluation of the exposure in Italian population. Journal of Agricultural and Food Chemistry, Easton, v.56, n.22. p.10611-10618, 2008. Disponível em: <http://dx.doi.org/ 10.1021/jf8016282>. Acesso em: 20 set. 2008. doi: 10.1021/ jf8016282.

BROWN, A.L. et al. DNA ploidy distribution in renal tumors induced in male rats by dietary ochratoxin. Experimental and Toxicologic Pathology, Jena, v.59, n.2, p.85-95, 2007. Disponível em: <http://dx.doi.org/10.1016/j.etp.2007.05.001>. Acesso em: 20 set. 2008. doi: 10.1016/j.etp.2007.05.001.

BURDASPAL, P.A.; LEGARDA, T.M. Ocratoxina a en vinos, mostos y zumos de uva elaborados en España y en otros paises europeos. Alimentaria, Madrid, v.299, p.107-113, 1999.

BURDASPAL, P.; LEGARDA, T. Occurrence of ochratoxin A in sweet wines produced in Spain and other countries. Food Additives and Contaminants, London, v.24, n.9, p.976986, 2007. Disponível em: <http://dx.doi.org/10.1080/ 02652030701311155>. Acesso em: 20 set. 2008. doi: 10.1080/ 02652030701311155 .

CABAÑES, F.J. et al. What is the source of ochratoxin A in wine? International Journal of Food Microbiology, Amsterdam, v.79, p.213-215, 2002. Disponível em: <http:// 
dx.doi.org/10.1016/S0168-1605(02)00087-9>. Acesso em: 20 set. 2008. doi: $10.1016 / \mathrm{S} 0168-1605(02) 00087-9$.

CHULZE et al. Occurrence of ochratoxin A in wine and ochratoxigenic mycoflora in grapes and dried vine fruits in South America. International Journal of Food Microbiology, Amsterdam, v.111, p.S5-S9, 2006. Disponível em: <http://dx.doi.org/10.1016/j.ijfoodmicro.2006.02.006>. Acesso em: 20 set. 2008. doi: 10.1016/ j.ijfoodmicro.2006.02.006.

COMMISSION OF THE EUROPEAN COMMUNITIES. Setting maximum levels for certain contaminants in foodstuffs, No $1881 / 2006$ of 19 December 2006. Official Journal of the European Union, Bruxelles, v.L364, p.5-24, 2006.

COZZI, G. et al. Effect of Lobesia botrana damages on black aspergilli rot and ochratoxin A content in grapes. International Journal of Food Microbiology, Amsterdam, v.111, p.88-92, 2006. Disponível em: <http://dx.doi.org/10.1016/10.1016/ j.ijfoodmicro.2006.03.012>. Acesso em: 20 set. 2008. doi: 10.1016/j.ijfoodmicro.2006.03.012.

DALL'ASTA, C. et al. The occurrence of ochratoxin A in blue cheese. Food Chemistry, London, v.106, n.2, p.729-734, 2008. Disponível em: <http://dx.doi.org/10.1016/j.foodchem.2007.06.049> Acesso em: 20 set. 2008. doi: 10.1016/j.foodchem.2007.06.049.

DRUSCH, S.; RAGAB, W.S.M. Mycotoxins in fruits, fruit juices and dried fruits. Journal of Food Protection, Des Moines, v.66, p.1514-1527, 2003.

ESTEBAN, A. et al. Effects of temperature and incubation time on production of ochratoxin A by black aspergilli. Research in Microbiology, Paris, v.155, p.861-866, 2004. Disponível em: $<$ http://dx.doi.org/10.1016/j.resmic.2004.07.002>. Acesso em: 20 set. 2008. doi: 10.1016/j.resmic.2004.07.002.

FERNANDES, A. et al. Fate of ochratoxin A during a vinification trial. Aspects of Applied Biology, Wellesbourne, v.68, p.73-80, 2003. Disponível em: <http://dx.doi.org/ 10.1021/jf061669d>. Acesso em: 20 set. 2008. doi: 10.1021/ jf061669d.

FILALI, A. et al. Ochratoxin A in beverages from Morocco: a preliminary survey. Food Additives and Contaminants, London, v.18, p.565-568, 2001. Disponível em: <http:// dx.doi.org/10.1080/02652030117365>. Acesso em: 20 set. 2008. doi: $10.1080 / 02652030117365$.

FINOLI, C. et al. Ochratoxin A occurrence in Italian wines of different origins. Rivista di Viticoltura e di Enologia, Treviso, v.57, p.63-77, 2004.

FLAJS, D. et al. ELISA and HPLC analysis of ochratoxin A in red wines of Croatia. Food Control, v. 20, n.6, p.590-592, 2009. Disponível em: <http://dx.doi.org/10.1016/j.foodcont.2008.08.021>. Acesso em: 20 set. 2008. doi: 10.1016/j.foodcont.2008.08.021.

GRAZIOLI, B. et al. The role of processing on ochratoxin A content in Italian must and wine: A study on naturally contaminated grapes. International Journal of Food Microbiology, Amsterdam, v.111, n.1, p.93-96, 2006. Disponível em: <http:// dx.doi.org/10.1016/j.ijfoodmicro.2006.01.045>. Acesso em: 20 set. 2008. doi: 10.1016/j.ijfoodmicro.2006.01.045.
GUZEV, L. et al. Occurrence of ochratoxin A producing fungi in wine and table grapes in Israel. International Journal of Food Microbiology, Amsterdam, v.111, p. S67-S71, 2006. Disponível em: <http://dx.doi.org/10.1016/j.ijfoodmicro.2006.03.003>. Acesso em: 20 set. 2008. doi: 10.1016/j.ijfoodmicro.2006.03.003.

GUZEV, L. et al. The effects of cold storage of table grapes, sulphur dioxide and ethanol on species of black Aspergillus producing ochratoxin A. International Journal of Food Science and Technology, Oxford, v.43, p.1187-1194, 2008. Disponível em: $<$ http://dx.doi.org/10.1111/j.1365-2621.2007.01589.x>. Acesso em: 20 set. 2008 . doi: 10.1111/j.1365-2621.2007.01589.x.

HASSEN, W. et al. Ochratoxin A and b2-microglobulinuria in healthy individuals and chronic interstitial nephropathy patients in the Centre of Tunisia: a hot spot of ochratoxin A exposure. Toxicology, Limerick, v.199, p.185-193, 2004. Disponível em: <http://dx.doi.org/10.1016/j.tox.2004.02.027>. Acesso em: 20 set. 2008 . doi: 10.1016/j.tox.2004.02.027.

HOCKING, A.D. et al. Occurrence of ochratoxin A in Australian wine. Australian Journal of Grape and Wine Research, Adelaide, v.9, p.72-78, 2003.

IARC (International Agency for Research on Cancer). Monographs on evaluation of carcinogenic risks to humans: some naturally occurring substances, food items and constituents, heterocyclic aromatic amines and mycotoxins. Lyon: IARC, 1993. 571p.

JECFA. Joint FAO/WHO Expert Committee on Food Additives. Safety evaluations on certain mycotoxins in food 2001 Acesso em 22 set. 2008. Online. Disponível em: http:// www.inchem.org/documents/jecfa/jecmono/v47je01.htm.

JORGENSEN, K.; JACOBSEN, J.S. Occurrence of ochratoxin A in Danish wheat and rye, 1992-99. Food Additives and Contaminants, London, v.19, n.12, p.1184-1189, 2002. Disponível em: <http://dx.doi.org/10.1080/0265203021000012376>. Acesso em: 20 set. 2008 . doi: 10.1080/0265203021000012376.

KARBANCIOLU-GULER, F.; HEPERKAN, D. Natural occurrence of ochratoxin A in dried figs. Analytica Chimica Acta, Amsterdam, v.617, n.1-2, p.32-36, 2008. Disponível em: <http://dx.doi.org/10.1016/j.aca.2008.01.009>. Acesso em: 20 set. 2008. doi: 10.1016/j.aca.2008.01.009.

KAWASHIMA, L.M. et al. Fumonisin B1 and ochratoxin A in beers made in Brazil. Ciência e Tecnologia de Alimentos, Campinas, v.27, n.2, p.317-323, 2007. Disponível em: <http:/ /dx.doi.org/10.1590/S0101-20612007000200019>. Acesso em: 20 set. 2008 . doi: 10.1590/S0101-20612007000200019.

KHOURY, A. et al. Occurrence of Ochratoxin A and Aflatoxin B1-producing fungi in lebanese grapes and Ochratoxin A content in musts and finished wines during 2004. Journal of Agricultural and Food Chemistry, Easton, v.54, n.23, p.8977-8982, 2006. Disponível em: <http://dx.doi.org/10.1021/jf062085e $>$. Acesso em: 20 set. 2008 . doi: 10.1021/jf062085e.

KHOURY, A. et al. Fungal contamination and Afaltoxin B1 and Ochratoxin A in Lebanese wine-grapes and musts. Food and Chemical Toxicology, Oxford, v.46, n.6, p.2244-2250, 2008. Disponível em: <http://dx.doi.org/10.1016/j.fct.2008.02.026>. Acesso em: 20 set. 2008. doi: 10.1016/j.fct.2008.02.026. 
LASRAM, S. et al. Occurrence of ochratoxigenic fungi and ochratoxin A in grapes from a Tunisian vineyard. International Journal of Food Microbiology, Amsterdam, v.114, n.3, p.376-379, 2007. Disponível em: <http://dx.doi.org/ 10.1016/j.ijfoodmicro.2006.09.027>. Acesso em: 20 set. 2008. doi: $10.1016 /$ j.ijfoodmicro.2006.09.027.

LASRAM, S. et al. Evolution of ochratoxin A content during red and rose vinification. Journal of the Science of Food and Agriculture, London, v.88, n.10, p.1696-1703, 2008. Disponível em: <http://dx.doi.org/10.1002/jsfa.3266>. Acesso em: 20 set. 2008 . doi: $10.1002 /$ jsfa. 3266 .

LATASTE, C. et al. Development of contamination by ochratoxin A: of the French vineyard. Progres Agricole et Viticole, Logroño, v.121, p.57-64, 2004.

LEONI, L.A.B. et al. Ochratoxin A in Brazilian roasted and instant coffees. Food Additives and Contaminants, London, v.17, n.10, p.867-870, 2000.

LEONG, S.L. et al. Occurrence of fruit rot fungi (Aspergillus section Nigri) on some drying varieties of irrigated grapes. Australian Journal of Grape and Wine Research, Adelaide, v.10, p.83-88, 2004.

LEONG, S.L. et al. Australian research on ochratoxigenic fungi and ochratoxin A. International Journal of Food Microbiology, Amsterdam, v.111, n.S1, p.S10-S17, 2006a. Disponível em: <http:/ /dx.doi.org/10.1016/j.ijfoodmicro.2006.02.005>. Acesso em: 20 set. 2008. doi: 10.1016/j.ijfoodmicro.2006.02.005.

LEONG, S.L. et al. Fate of ochratoxin A during vinification of Semillon and Shiraz grapes. Journal of Agricultural and Food Chemistry, Easton, v.54, p.6460-6464, 2006b. Disponível em: $<\mathrm{http}$ //dx.doi.org/10.1021/jf061669d $>$. Acesso em: 20 set. 2008 . doi: $10.1021 /$ jf061669d.

LEONG, S.L. et al. Ochratoxin A-producing Aspergilli in Vietnamese green coffee beans. Letters in Applied Microbiology, Oxford, v.45, n.3, p.301-306, 2007. Disponível em: <http://dx.doi.org/10.1111/j.1472-765X.2007.02189.x>. Acesso em: 20 set. 2008. doi: 10.1111/j.1472765X.2007.02189.x.

LINO, C.M. et al. Levels of ochratoxin A in serum from urban and rural Portuguese populations and estimation of exposure degree. Food and Chemical Toxicology, Oxford, v.46, n.3, p.879-885, 2008. Disponível em: <http://dx.doi.org/10.1016/ j.fct.2007.10.012>. Acesso em: 20 set. 2008. doi: 10.1016/ j.fet.2007.10.012.

MAGNOLI, C. et al. Mycoflora and ochratoxin-producing strains of Aspergillus section Nigri in wine grapes in Argentina. Letters in Applied Microbiology, Oxford, v.37, p.179-184, 2003. Disponível em: <http://dx.doi.org/10.1046/j.1472765X.2003.01376.x>. Acesso em: 20 set. 2008. doi: 10.1046/ j.1472-765X.2003.01376.x.

MAGNOLI, C. et al. Occurrence of ochratoxin $A$ and ochratoxigenic mycoflora in corn and corn based foods and feeds in some South American countries. Mycopathologia, Den Haag v.163, n.5, p.249-260, 2007. Disponível em: <http://dx.doi.org/10.1007/s1 1046-007-9005-z>. Acesso em: 20 set. 2008. doi: 10.1007/s11046-007-9005-z.
MARKAKI, P. et al. Determination of Ochratoxin A in red wine and vinegar by immunoaffinity high-pressure liquid chromatography. Journal of Food Protection, Des Moines, v.64, p.533-537, 2001.

MIRAGLIA, M.; BRERA, C. Assessment of dietary intake of Ochratoxin A by the population of EU Member States. Reports on Tasks for Scientific Cooperation.Directorate General of Health and Consumer Protection. Brussels: European Commission, 2002. Acesso em 4 jan. 2009. Online. Disponível em: http://ec.europa.eu/food/fs/scoop/3.2.7_en.

MITCHELL, D. et al. Water and temperature relations of growth and ochratoxin A production by Aspergillus carbonarius strains from grapes in Europe and Israel. Journal of Applied Microbiology, Oxford, v.97, p.439-445, 2004. Disponível em: <http://dx.doi.org/10.1111/j.1365-2672.2004.02321.x>. Acesso em: 20 set. 2008. doi: 10.1111/j.13652672.2004.02321.x.

MOUNJOUENPOU, P. et al. Filamentous fungi producing ochratoxin A during cocoa processing in Cameroon. International Journal of Food Microbiology, Amsterdam, v.121, n.2, p.234-241, 2008. Disponível em: <http://dx.doi.org/ 10.1016/j.ijfoodmicro.2007.11.017>. Acesso em: 20 set. 2008. doi: $10.1016 /$ j.ijfoodmicro.2007.11.017.

OLIVERI, C. et al. A polyphasic approach to the identification of ochratoxin A-producing black Aspergillus isolates from vineyards in Sicily. International Journal of Food Microbiology, Amsterdam, v.127, n.1-2, p.147-154, 2008. Disponível em: <http:/ /dx.doi.org/10.1016/j.ijfoodmicro.2008.06.021>. Acesso em: 20 set. 2008. doi: 10.1016/j.ijfoodmicro.2008.06.021.

OTTENEDER, H.; MAJERUS, P. Occurrence of ochratoxin A (OTA) in wines: influence of the type of wine and its geographical origin. Food Additives and Contaminants, London, v.17, p.793-798, 2000. Disponível em: $<$ http:// dx.doi.org/10.1080/026520300415345>. Acesso em: 20 set. 2008. doi: $10.1080 / 026520300415345$.

PALMA, N. et al. Ochratoxin A-induced mutagenesis in mammalian cells is consistent with the production of oxidative stress. Chemical Research in Toxicology, Washington, v.20, n.7, p.1031-1037, 2007. Disponível em: <http://dx.doi.org/ 10.1021/tx700027j>. Acesso em: 20 set. 2008. doi: 10.1021/ $\operatorname{tx} 700027 \mathrm{j}$.

PERRONE, G. et al. Positive correlation between high levels of ochratoxin A and resveratrol related compounds in red wines. Journal of Agricultural and Food Chemistry, Easton, v.55, p.6807-6812, 2007. Disponível em: <http://dx.doi.org/ 10.1021/jf0709931>. Acesso em: 10 set. 2008. doi: 10.1021/ jf0709931.

PERRONE, G. et al. Aspergillus uvarum sp., an uniseriate black Aspergillus species isolated from grapes in Europe. International Journal of Systematic and Evolutionary Microbiology, Reading, v.58, p.1032-1039, 2008. Disponível em: <http://dx.doi.org/10.1099/ijs.0.65463-0>. Acesso em: 20 set. 2008. doi: 10.1099/ijs.0.65463-0.

PIETRI, A. et al. Occurrence of ochratoxin A in Italian wines. Food Additives and Contaminants, London, v.18, p.647654, 2001. Disponível em: <http://dx.doi.org/10.1080/ 02652030119480>. Acesso em: 20 set. 2008. doi: 10.1080/ 02652030119480 .

Ciência Rural, v.39, n.8, nov, 2009. 
RIBA, A. et al. Mycoflora and ochratoxin A producing strains of Aspergillus in Algerian wheat. International Journal of Food Microbiology, Amsterdam, v.122, n.1-2, p.85-92, 2008. Disponível em: <http://dx.doi.org/10.1016/j.ijfoodmicro.2007.11.057>. Acesso em: 10 set. 2008. doi: 10.1016/j.ijfoodmicro.2007.11.057.

ROSA, R.C.A. et al. Potential ochratoxin A producers from wine grapes in Argentina and Brazil. Food Additives and Contaminants, London, v.19, p.408-414, 2002. Disponível em: <http://dx.doi.org/10.1080/02652030110092748>. Acesso em: 20 set. 2008 . doi: 10.1080/02652030110092748.

ROSA, R.C.A. et al. Occurrence of ochratoxin A in wine and grape juice marketed in Rio de Janeiro, Brazil. Food Additives and Contaminants, London, v.21, p.358-364, 2004. Disponível em: $<$ http://dx.doi.org/10.1080/02652030310001639549>. Acesso em: 20 set. 2008 . doi: 10.1080/02652030310001639549.

ROSSIELLO, M.R. et al. Ochratoxin A inhibits the production of tissue factor and plasminogen activator inhibitor-2 by human blood mononuclear cells: Another potential mechanism of immune-suppression. Toxicology and Applied Pharmacology, San Diego, v.229, p.227-231, 2008. Disponível em: <http:// dx.doi.org/10.1016/j.taap.2008.01.004>. Acesso em: 12 set. 2008. doi: 10.1016/j.taap.2008.01.004.

SAGE, L. et al. Fungal flora and ochratoxin A production in grapes and musts from France. Journal of Agricultural and Food Chemistry, Easton, v.50, p.1306-1311, 2002. Disponível em: <http://dx.doi.org/10.1021/jf011015z>. Acesso em: 20 set. 2008 . doi: 10.1021/jf011015z.

SANGARE-TIGORI, B. et al. Ochratoxin A in human blood in Abidjan, Côte d'Ivoire. Toxicon, Elmsford, v.47, n.8, p.894 900, 2006. Disponível em: <http://dx.doi.org/10.1016/ j.toxicon.2006.03.001>. Acesso em: 20 set. 2008. doi: $10.1016 /$ j.toxicon.2006.03.001.

SAVA, V. et al. Acute neurotoxic effects of the fungal metabolite ochratoxin-A. Neurotoxicology, Little Rock, n.27, v.1, p.8292, 2006. Disponível em: <http://dx.doi.org/10.1016/ j.neuro.2005.07.004>. Acesso em: 20 set. 2008. doi: 10.1016/ j.neuro.2005.07.004.

SEKIYAMA, B.L. et al. Aflatoxins, ochratoxin A and zearalenone in maize-based food products. Brazilian Journal of Microbiology, v.36, n.3, p. 289-294, 2005. Disponível em: <http://dx.doi.org/10.1590/S1517-83822005000300016>. Acesso em: 10 set. 2008. doi: 10.1590/S151783822005000300016 .

SERRA, R. et al. Black Aspergillus species as ochratoxin A producers in Portuguese wine grapes. International Journal of Food Microbiology, Amsterdam, v.88, p.63-68, 2003. Disponível em:<http://dx.doi.org/10.1016/S0168-1605(03)00085-0>. Acesso em: 20 set. 2008. doi: 10.1016/S0168-1605(03)00085-0.

SERRA, R. et al. Ochratoxin A occurrence and formation in Portuguese wine grapes at various stages of maturation. International Journal of Food Microbiology, Amsterdam, v.111, p.S35-S39, 2006. Disponível em: <http://dx.doi.org/ 10.1016/j.ijfoodmicro.2006.03.007>. Acesso em: 20 set. 2008. doi: $10.1016 /$ j.ijfoodmicro.2006.03.007.

SHEPHARD, G.S. et al. Quantitation of ochratoxin A in South African wines. Journal of Agricultural and Food Chemistry, Easton, v.51, p.1102-1106, 2003. Disponível em: <http://dx.doi.org/10.1021/jf0259866>. Acesso em: 20 set. 2008. doi: 10.1021/jf0259866. 